\title{
Interneuron Transplantation Rescues Social Behavior Deficits without Restoring Wild-Type Physiology in a Mouse Model of Autism with Excessive Synaptic Inhibition
}

\author{
Derek G. Southwell, ${ }^{1,2,3}$ Helia Seifikar, ${ }^{2,3,4,5}$ @Ruchi Malik, ${ }^{2,3,4,5}$ Karen Lavi, ${ }^{2,3,4,5}$ Daniel Vogt, ${ }^{2,3,5}$ \\ (D) John L. Rubenstein, ${ }^{2,3,5}$ and DVikaas S. Sohal $2,3,4,5$ \\ ${ }^{1}$ Department of Neurological Surgery, ${ }^{2}$ Weill Institute for Neurosciences, ${ }^{3}$ Kavli Institute for Fundamental Neuroscience, ${ }^{4}$ Sloan Swartz Center for \\ Theoretical Neurobiology, and ${ }^{5}$ Department of Psychiatry, University of California, San Francisco, San Francisco, California 94143
}

\begin{abstract}
Manipulations that enhance GABAergic inhibition have been associated with improved behavioral phenotypes in autism models, suggesting that autism may be treated by correcting underlying deficits of inhibition. Interneuron transplantation is a method for increasing recipient synaptic inhibition, and it has been considered a prospective therapy for conditions marked by deficient inhibition, including neuropsychiatric disorders. It is unknown, however, whether interneuron transplantation may be therapeutically effective only for conditions marked by reduced inhibition, and it is also unclear whether transplantation improves behavioral phenotypes solely by normalizing underlying circuit defects. To address these questions, we studied the effects of interneuron transplantation in male and female mice lacking the autism-associated gene, Pten, in GABAergic interneurons. Pten mutant mice exhibit social behavior deficits, elevated synaptic inhibition in prefrontal cortex, abnormal baseline and social interaction-evoked electroencephalogram (EEG) signals, and an altered composition of cortical interneuron subtypes. Transplantation of wild-type embryonic interneurons from the medial ganglionic eminence into the prefrontal cortex of neonatal Pten mutants rescued social behavior despite exacerbating excessive levels of synaptic inhibition. Furthermore, transplantation did not normalize recipient EEG signals measured during baseline states. Interneuron transplantation can thus correct behavioral deficits even when those deficits are associated with elevated synaptic inhibition. Moreover, transplantation does not exert therapeutic effects solely by restoring wild-type circuit states. Our findings indicate that interneuron transplantation could offer a novel cell-based approach to autism treatment while challenging assumptions that effective therapies must reverse underlying circuit defects.
\end{abstract}

Key words: autism; excitatory/inhibitory balance; interneuron transplantation

\section{Significance Statement}

Imbalances between neural excitation and inhibition are hypothesized to contribute to the pathophysiology of autism. Interneuron transplantation is a method for altering recipient inhibition, and it has been considered a prospective therapy for neuropsychiatric disorders, including autism. Here we examined the behavioral and physiological effects of interneuron transplantation in a mouse genetic model of autism. They demonstrate that transplantation rescues recipient social interaction deficits without correcting a common measure of recipient inhibition, or circuit-level physiological measures. These findings demonstrate that interneuron transplantation can exert therapeutic behavioral effects without necessarily restoring wild-type circuit states, while highlighting the potential of interneuron transplantation as an autism therapy.

\section{Introduction}

The dysfunction of GABAergic interneurons, or "interneuronopathy", has been implicated in the pathophysiology of autism spec- trum disorder (ASD; Lewis et al., 2005; Marín, 2012). As such, treatments that correct or compensate for interneuron dysfunction may represent a novel therapeutic approach to some forms of 
ASD. Embryonic interneuron transplantation is a method for modifying recipient neural circuit function, and it has been considered a prospective treatment for neuropsychiatric disorders including ASD (Southwell et al., 2014; Tyson and Anderson, 2014; Spatazza et al., 2017). When transplanted from the embryonic ventral forebrain, immature interneurons disperse and survive in the postnatal cerebral cortex where they differentiate into mature GABAergic interneurons, form functional synapses, increase recipient synaptic inhibition, and modify plasticity (Alvarez-Dolado et al., 2006; Baraban et al., 2009; Southwell et al., 2010; Davis et al., 2015; Howard and Baraban, 2016; Hsieh and Baraban, 2017; Larimer et al., 2017). Because interneuron transplantation robustly increases synaptic measures of inhibition, it is assumed that transplantation may be most beneficial for conditions marked by diminished inhibition, where it is expected to exert therapeutic effects by restoring normal levels of inhibitory signaling.

Autism therapies have been conceptualized by studies that (1) identify a physiologic abnormality in a mouse model, and (2) demonstrate behavioral rescue following experimental reversal of the physiologic abnormality. These studies typically involved models marked by excessive excitation or deficient inhibition, and, as such, they examined therapeutic manipulations intended to enhance inhibitory signaling. These include (1) stimulating inhibitory neurons in CNTNAP2 mutant mice (Selimbeyoglu et al., 2017), Neuroligin-3 knock-in mice (Cao et al., 2018), and in an optogenetic model of elevated excitation (Yizhar et al., 2011); and (2) administering benzodiazepines to $S c n 1 A^{+/-}$mice (Han et al., 2012), BTBR inbred mice (Gogolla et al., 2014), and Arid $1 b^{+/-}$mice (Jung et al., 2017). In each of these studies, it was hypothesized that, because (1) synaptic inhibition was initially inadequate, and (2) behavior was normalized following a manipulation that enhanced inhibition, then autism phenotypes could be corrected by increasing overall inhibition to restore a wildtype-like circuit state. However, it is possible that these manipulations, and others such as interneuron transplantation, could alternatively or additionally elicit more complex circuit changes, modifying behavior through mechanisms that do not necessarily involve normalization of underlying measures of circuit physiology, such as synaptic inhibition. These alternatives have important implications for the development of autism therapies and for the application of interneuron transplantation as a therapeutic strategy in general.

To address these questions, we have studied the effects of interneuron transplantation in a mouse model of autism that exhibits excessive synaptic inhibition: Nkx2.1-Cre; Pten conditional knock-out mice. We previously showed that, in Nkx2.1-Cre; Pten conditional knock-out mice (hereafter also referred to as "Pten mice"), disruption of Pten, a phosphatase implicated in human ASD (Roak et al., 2012; De Rubeis et al., 2014; Iossifov et al., 2015; Geisheker et al., 2017), alters the subtype composition of interneurons derived from the medial ganglionic eminence [somatostatin (SST)- and parvalbumin (PV)-expressing subpopulations] and increases inhibitory signaling onto excitatory neurons in prefrontal cortex (PFC; Vogt et al., 2015). Furthermore, Pten mice exhibit altered oscillatory activity on EEG and deficits in social behavior.

Correspondence should be addressed to Derek G. Southwell at derek.southwell@duke.edu or Vikaas S. Sohal at vikaas.sohal@ucsf.edu.

D. G. Southwell's present address: Department of Neurosurgery and Graduate Program in Neurobiology at Duke University, Durham, NC 27710.

D. Vogt's present address: Departments of Pediatrics and Human Development at Michigan State University, Grand Rapids, MI 49503.

https://doi.org/10.1523/JNEUROSCl.1063-19.2019

Copyright $\odot 2020$ the authors
By studying the effects of interneuron transplantation in Pten mice, we demonstrate that transplantation can normalize ASD social behavior phenotypes, and, moreover, it can do so even in a recipient background marked by excessive, rather than deficient, inhibition. Surprisingly, these behavioral improvements occur even though recipient circuit physiology is not normalized by transplantation, suggesting that transplantation may yield therapeutic effects by creating a new circuit state, rather than restoring a wild-type one.

\section{Materials and Methods}

Experimental design and statistical analyses. The primary objective of this study was to determine the behavioral and physiologic effects of interneuron transplantation in a mouse model of ASD. Secondarily, we also sought to compare the effects of transplantation between ASD-like recipient mice and wild-type mice. We performed interneuron transplantation into Pten mice, an ASD model in which Pten, a high-confidence autism disease gene (Roak et al., 2012; De Rubeis et al., 2014; Iossifov et al., 2015; Geisheker et al., 2017), is disrupted in interneurons derived from the medial ganglionic eminence (Vogt et al., 2015). Our laboratory has previously characterized this mutant mouse model and noted robust histologic, physiologic, and behavioral phenotypes, some of which resemble features of human ASD (Vogt et al., 2015). In a separate series of experiments, we also assessed the behavioral and physiologic effects of interneuron transplantation in wild-type CD-1 mice that underwent interneuron transplantation during neonatal stages. This allowed us to compare the effects of transplantation between Pten mice and wild-type mice, and thereby ascertain potential relationships between the recipient background, or neural circuit function, and transplant effect.

Donor cells were obtained from embryonic mice that expressed green fluorescent protein (GFP; 53), and injected bilaterally into the PFC of neonatal recipient mice. Vehicle injections, without cells, were performed on control animals. Behavioral, physiologic, and histologic studies of recipient mice were performed at $\sim 7$ weeks after transplantation, $\sim$ postnatal day $(\mathrm{P}) 50$. Electroencephalography electrodes were surgically implanted into the PFC 1 week before behavioral analysis to allow the recipient animals sufficient recovery time before behavioral studies were performed. We studied recipient behavior at P50 because we have observed that Pten mice exhibit histologic, physiologic, and behavioral abnormalities by this time point (Vogt et al., 2015). Additionally, the transplanted interneuron population is expected to stabilize in size and reach physiologic maturity in the recipient by this time point (AlvarezDolado et al., 2006; Southwell et al., 2012; Howard and Baraban, 2016; Larimer et al., 2017). Electroencephalography was used to perform in vivo measurements of neural oscillations during baseline states. We also performed slice electrophysiology in vitro to examine synaptic measures of inhibition 7 weeks after transplantation into Pten mice. Finally, to confirm the presence and molecular phenotypes of transplanted cells, histology was performed on brain slices from recipient mice that underwent EEG and behavioral studies.

Power calculations to determine animal group size were based on previous studies describing Nkx2.1-Cre; Pten cKO mice (Vogt et al., 2015) and recipient responses to transplantation (Alvarez-Dolado et al., 2006; Baraban et al., 2009; Tanaka et al., 2011; Howard et al., 2014). Animals were housed and handled in a similar manner throughout the course of the study. For behavioral experiments, we did not define outliers or exclude mice from analysis. During behavioral studies, investigators were blinded to animal genotype and treatment.

Data were analyzed as specified using Prism (GraphPad). Equality of variance for grouped electrophysiology data were tested using the Bartlett test. Normality of data were tested using the Shapiro-Wilk test, Lilliefors test, and Anderson-Darling test; data with a non-normal distribution were analyzed using the Mann-Whitney test, and data with a normal distribution were analyzed using the Student's $t$ test. Group data are presented as mean \pm standard error of measurement (SEM). For each experiment, sample numbers are presented in the corresponding Results sections.

Experimental animals. All experiments were performed in accordance with the guidelines of the Administrative Panels on Laboratory Animal 
Care at the University of California, San Francisco, and with the NIH Guide for the Care and Use of Laboratory Animals. Mice were housed in an environment with a regular light/dark cycle (light period: 7:00 A.M. to 7:00 P.M.). Male and female mice were randomly assigned to experimental groups. Nkx2.1-Cre; Pten cKO recipient mice were generated from transgenic mouse lines that have been described previously: Nkx2.1-Cre (Xu et al., 2008), Pten flox (Suzuki et al., 2001), and Ai14 Cre-reporter (Madisen et al., 2010). Nkx2.1-Cre; Pten cKO mice were first maintained on a mixed CD-1 and C57BL/6J background; the mice used in this study were generated from animals that had been back-crossed to wild-type CD-1 mice for at least four generations. Resultant mice from these crosses were randomly assigned to experimental groups. Wild-type CD-1 neonatal recipient mice, wild-type $\mathrm{CD}-1$ adult breeder mice, and wildtype C57BL/6 juvenile mice (used for social interaction task) were purchased from Envigo. Fluorescent donor embryos were generated by crossing homozygous, $\beta$-actin:GFP mice (GFP mice; Hadjantonakis et al., 1998) to wild-type CD-1 mice. GFP mice were maintained on a mixed CD-1 and C57BL/6J background. Timed matings were performed to generate embryonic donors, and dams were examined for vaginal plugs each morning. If a plug was noted, the embryonic litter was considered to be of gestational age embryonic day (E)0.5. Mice were fed ad libitum and housed in standard lighting conditions ( $12 \mathrm{~h}$ light/dark cycle).

Interneuron transplantation. Embryonic donor medial ganglionic eminence (MGE) was obtained as previously described (Southwell et al., 2010). MGE tissue was explanted from E13.5 heterozygous GFP embryos and placed in chilled Liebovitz's L-15 medium containing $100 \mathrm{U} / \mathrm{ml}$ DNase I (New England Biolabs). The entire dorsal-ventral extent of the MGE was harvested from each donor. Pooled MGE explants, which were typically collected from 8 to 12 donors, were mechanically dissociated by trituration, and the resultant cell suspension was concentrated by centrifugation for $3 \mathrm{~min}$ at $\sim 800 \times \mathrm{g}$. Concentrated cells (density 800-1000 cells/nl) were loaded into beveled glass needles (inner diameter, $\sim 60$ $\mu \mathrm{m}$ ) attached to a hydraulic micromanipulator (Narishige).

Neonatal recipient mice (P1-P3) were anesthetized by hypothermia and placed in a custom head mold for injections. Cells were injected into the bilateral PFC ( $100 \mathrm{nl}$ per hemisphere) of recipient mice using coordinates that were described previously (Tanaka et al., 2011). Control, sham injections were performed using L-15 medium with DNase. After cell transplantation or sham injection, recipient mice were placed on a warm heating pad and returned to their mothers. Each transplant recipient group contained subsets of mice that received transplants from distinct donor pools. In some cases the different transplant recipient groups (Pten mutant and wild-type CD-1) received transplants from the same donor pools.

EEG surgery. Six weeks after cell transplantation (P42-P45), recipient mice underwent PFC EEG implantation. Mice were anesthetized with isoflurane and fixed in a stereotaxis (Kopf Instruments). Stainless steel EEG skull screws (Pinnacle Technology) were implanted over the bilateral PFC at coordinates $1.7 \mathrm{~mm}$ anterior and $\pm 0.5 \mathrm{~mm}$ lateral to the bregma. A ground electrode was implanted over the cerebellum at coordinates $5 \mathrm{~mm}$ posterior and $0 \mathrm{~mm}$ lateral to the bregma. The EEG screws were connected to a four-channel EEG/EMG head mount (Pinnacle Technology) using conductive wires. The EEG electrodes and head mounts were fixed to the skull using dental cement.

EEG recordings and analysis. EEG recordings were performed 1 week after EEG implantation, at a time point $\sim 7$ weeks after interneuron transplantation (or sham injection). Mice were connected to the EEG preamplifier and placed in their home cage. Baseline EEG activity was then recorded for $10 \mathrm{~min}$ in the absence of other mice. A time-locked video EEG monitoring system (Pinnacle Technology) was used to record EEG signals. Signals were recorded at a sampling rate of $2000 \mathrm{~Hz}$, with low- and high-pass filters of 0.5 and $200 \mathrm{~Hz}$, respectively. EEG recordings were analyzed using the power spectral density output from the spectrogram function in MATLAB (MathWorks) with a window size of $2^{11}$ and no overlap. Power spectra from 0 to $150 \mathrm{~Hz}$ were quantified for each mouse during the $10 \mathrm{~min}$ baseline period. To compare baseline power in particular frequency bands between genotypes, we first normalized, for each animal, power in each band by the total power, then used $t$ tests.
Animals with EEG traces that exhibited large $60 \mathrm{~Hz}$ noise artifacts were excluded from EEG analysis.

Behavioral assays. Behavioral assays were performed 1 week after EEG implantation, at a time point $\sim 7$ weeks after interneuron transplantation (or sham injection). Assays were performed during the first half of the animals' light cycle, and they were conducted in an isolated behavior room remote from their housing area. Animals were handled minimally before behavioral testing, and they were allowed to habituate to the behavior room for $1 \mathrm{~h}$ before the initiation of behavioral testing. The experimenter was absent during this habituation period. All animals then underwent the following sequence of behavioral studies (from first to last): social interaction test, novel object test, elevated plus maze, and open-field test. The order of behavioral tests did not vary between animals. Each animal underwent testing once. To avoid disturbing experimental mice during the behavioral assays, the experimenter was absent from the testing area during each assay. When scoring behavior, experimenters were blind to animal Pten genotype and treatment (cell transplant vs sham treatment).

Social interaction test. After the baseline EEG recording, a novel juvenile mouse (age P20-P30, of the same sex as the experimental mouse) was then placed into the home cage of the experimental mouse, as previously described (Vogt et al., 2015). The experimental mouse was allowed to freely interact with the novel juvenile mouse for $5 \mathrm{~min}$. Video recordings were subsequently reviewed to identify periods of social interaction (defined by period in which the experimental mouse sniffed, closely followed, or groomed the foreign mouse). The total length of the social interaction periods was measured.

Novel object test. Following the social interaction test, the novel mouse was removed, and the experimental mouse was left alone in its home cage. As described in previous studies (Vogt et al., 2015; Selimbeyoglu et al., 2017), a novel object (a blue disc, $2 \mathrm{~cm}$ in diameter and $1 \mathrm{~cm}$ in height) was then placed at one end of the home cage for $5 \mathrm{~min}$. Video recordings were analyzed to quantify the length of time the experimental mouse spent investigating the novel object (defined as sniffing the novel object or visually examining the novel object at a distance of $<2.5 \mathrm{~cm}$ ).

Elevated plus maze. Following the novel object test, the experimental mouse was placed on a four-armed + -shaped platform that was elevated $40 \mathrm{~cm}$ from the floor. Each arm of the platform was equal in size $(30 \mathrm{~cm}$ long by $5 \mathrm{~cm}$ wide), and oriented at a $90^{\circ}$ angle from the neighboring arms. The four arms intersected at a center area $(5 \times 5 \mathrm{~cm}$ wide $)$. Two of the arms, which were at $18^{\circ}$ from one another, were open (height of open arm walls, $0.5 \mathrm{~cm})$, whereas the other two arms $\left(180^{\circ}\right.$ from each other, and $90^{\circ}$ from the closed arms) were closed (height of walls, $15 \mathrm{~cm}$ ). The experimental mouse was placed in the center area and allowed to explore the platform for $10 \mathrm{~min}$. The experimental mouse's movements were recorded and analyzed by a video tracking system (ANY-maze) to quantify the number of entries into the open arms of the platform, as well as the amount of time spent in the open arms. The elevated plus maze was cleaned with $70 \%$ ethanol between assays of different animals.

Open-field test. Following the elevated plus maze test, the experimental mouse was placed in the center of an empty open field $(50 \times 50 \mathrm{~cm}$ wide, with walls $20 \mathrm{~cm}$ high). The experimental mouse was allowed to explore the open field for $10 \mathrm{~min}$, while its trajectory was recorded and analyzed by the video tracking system (ANY-maze) to quantify the time the animal spent and the distance it traveled in both the center (a $25 \times 25 \mathrm{~cm}$ area in center of the open field) and the periphery of the open field (all area outside the open-field center).

Slice preparation for intracellular electrophysiological recordings. A subset of mice that did not undergo EEG placement or behavioral assays was used to study cellular electrophysiology $\sim 7$ weeks after transplantation (or sham injection). Mice were anesthetized with an intraperitoneal injection of Euthasol and transcardially perfused with an ice-cold cutting solution that contained the following (in $\mathrm{mM}$ ): 210 sucrose, $2.5 \mathrm{KCl}, 1.25$ $\mathrm{NaH}_{2} \mathrm{PO}_{4}, 25 \mathrm{NaHCO}_{3}, 0.5 \mathrm{CaCl}_{2}, 7 \mathrm{MgCl}_{2}$, and 7 dextrose. Acute coronal slices (250 $\mu \mathrm{m}$ thick) containing the prelimbic and infralimbic regions of the medial PFC were obtained. Slices were allowed to recover at $34^{\circ} \mathrm{C}$ for $30 \mathrm{~min}$ followed by $30 \mathrm{~min}$ recovery at room temperature in a holding solution that contained the following (in $\mathrm{mm}$ ): $125 \mathrm{NaCl}, 2.5$ 


\section{P1-3: Interneuron Precursor Transplantation}

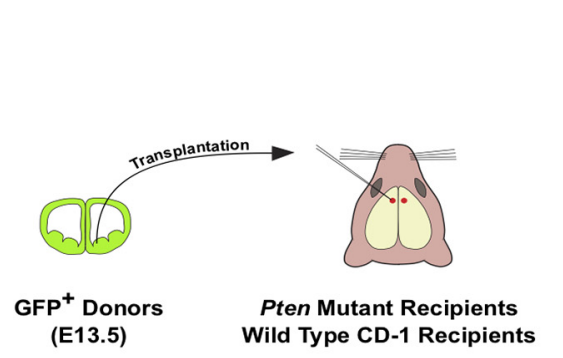

P50: In Vitro \& In Vivo Physiology, Behavioral Assays, Histology
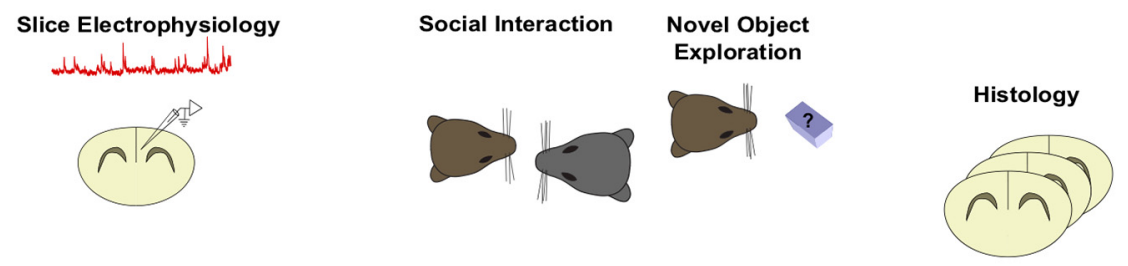

Open Field
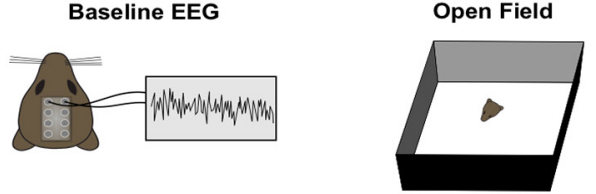

Elevated Plus Maze

Figure 1. Schematic of experimental design. Interneuron precursors were transplanted from the MGE of wild-type donors (E13.5) to the PFC of neonatal Pten mice or wild-type CD-1 mice. Seven weeks after transplantation, recipient brains were prepared for in vitro slice physiology. Recipient mice also underwent EEG recordings and behavioral assays (social interaction, novel object exploration, open-field exploration, and elevated plus maze exploration). Following behavioral assays, histology was performed to confirm the presence of transplanted interneurons and characterize the subtype composition of the transplanted interneurons.

$\mathrm{KCl}, 1.25 \mathrm{NaH}_{2} \mathrm{PO}_{4}, 25 \mathrm{NaHCO}_{3}, 2 \mathrm{CaCl}_{2}, 2 \mathrm{MgCl}_{2}, 12.5$ dextrose, 1.3 ascorbic acid, and 3 sodium pyruvate.

Whole-cell patch-clamp recordings. Whole-cell voltage-clamp recordings were obtained from submerged slices perfused in a heated $\left(32-34^{\circ} \mathrm{C}\right)$ recording solution (bubbled with $95 \% \mathrm{O}_{2} / 5 \% \mathrm{CO}_{2}, \mathrm{pH} \sim 7.4$ ) that contained the following (in mM): $125 \mathrm{NaCl}, 3 \mathrm{KCl}, 1.25 \mathrm{NaH}_{2} \mathrm{PO}_{4}, 25$ $\mathrm{NaHCO}_{3}, 2 \mathrm{CaCl}_{2}, 1 \mathrm{MgCl}_{2}$, and 12.5 dextrose. Slices were visualized using DIC optics fitted with a $40 \times$ water-immersion objective and fluorescent filters (BX51WI, Olympus Microscopy). Layer 2/3 pyramidal neurons in the medial PFC were identified by morphology (non-fluorescent cells with large, pyramidal soma), whereas layer $2 / 3 \mathrm{Nk} \times 2.1$-Cre; Pten cKO native interneurons and layer $2 / 3 \beta$-actin:GFP transplanted interneurons were identified by red (tdtomato) and green fluorescence, respectively. For all interneuron transplant recipients, transplanted cells were identified by imaging in the recorded field.

Somatic voltage-clamp recordings were obtained using patch pipettes (2-4 M $\Omega$ ) filled with an internal solution containing the following (in mM): 130 Cs-methanesulfonate, $10 \mathrm{CsCl}, 10 \mathrm{HEPES}, 4 \mathrm{NaCl}, 7$ phosphocreatine, $0.3 \mathrm{Na}-\mathrm{GTP}, 4 \mathrm{Mg}-\mathrm{ATP}$, and $2 \mathrm{QX} 314-\mathrm{Br}, \mathrm{pH} \sim 7.3$ adjusted with $\mathrm{CsOH}$. Data were acquired using MultiClamp 700B amplifier (Molecular Devices). Upon successful transition to the whole-cell configuration, a neuron was given at least $5 \mathrm{~min}$ to stabilize before data were collected. Spontaneous inhibitory currents were recorded for $5 \mathrm{~min}$ with neurons voltage-clamped at $10 \mathrm{mV}$. Series resistance was monitored throughout the recording and was typically $15-20 \mathrm{M} \Omega$. Recordings were discarded if series resistance was $>25 \mathrm{M} \Omega$. Spontaneous inhibitory currents were analyzed off-line using Clampfit (pClamp, Molecular Devices) event detection.

Slice preparation for histology. Mice were perfused with PBS and 4\% paraformaldehyde. Brains were then removed, postfixed overnight in $4 \%$ paraformaldehyde, and cryoprotected in $25 \%$ sucrose. Brains were frozen and cut into coronal sections ( $40 \mu \mathrm{m}$ thickness) with a frozen sliding microtome (Leica Biosystems).

Fluorescent immunostaining. Brain slices were blocked for $1-2 \mathrm{~h}$ at room temperature in a solution of 5\% bovine serum albumin (BSA) and $0.3 \%$ Triton X-100 in PBS. Slices were washed using PBS and incubated in antibody solutions (2.5\% BSA, $0.3 \%$ Triton X-100 in PBS) for $2-3 \mathrm{~h}$ at room temperature. Immunostaining was performed with the following primary antibodies: chicken anti-GFP (1:500; Aves Labs), mouse anti-PV (1:500; MilliporeSigma), rat anti-SST (1:200; MilliporeSigma), and mouse anti-Reelin (1:500; MilliporeSigma). The following secondary antibodies (1:300 dilution) were used for fluorescence labeling: AlexaFluor 488 goat anti-chicken, AlexaFluor 647 goat anti-mouse, and AlexaFluor 647 goat anti-rat (ThermoFisher Scientific). Slices were mounted on glass slides and coverslipped.

Histologic imaging and quantification of marker expression. Transplanted GFP + cells exhibited morphologies characteristic of interneu- rons. Transplanted interneurons were identified in slices from all animals that comprised the transplant-recipient groups. For quantification of marker expression in the transplanted cell population, fluorescent images were obtained using a digital camera (Photometrics) mounted on a conventional wide-field optical microscope with a $10 \times$ objective (Nikon Instruments). Images were obtained using appropriate filter settings to depict the transplanted cellular population, and then images were obtained to individually visualize each molecular marker of interest. Transplanted interneurons were analyzed in all layers of the recipient PFC. The fraction of transplanted cells expressing a given marker was determined by identifying GFP-expressing transplanted cells, then assessing for expression of the marker of interest (PV, SST, or Reelin) in the corresponding image obtained with the appropriate filter settings.

Histologic images (flattened $Z$-series of 15 confocal slices, $2 \mu \mathrm{m}$ thick) were obtained using a confocal microscope (Leica Microsystems).

\section{Results}

Interneuron transplantation rescues social interaction deficits in Pten mice

We bilaterally injected wild-type immature interneurons from the E13.5 MGE into the PFC of P1-P3 Pten mice. For controls, we performed sham (acellular vehicle) injections into the PFC of Pten mice and wild-type littermates (Fig. 1). The PFC was chosen as the transplantation site because prior studies have implicated prefrontal excitatory inhibitory balance in the regulation of social behavior (Yizhar et al., 2011; Selimbeyoglu et al., 2017). Seven weeks after transplantation, when transplanted MGE cells are expected to have differentiated into mature interneurons (AlvarezDolado et al., 2006; Southwell et al., 2012; Howard and Baraban, 2016; Larimer et al., 2017), we assessed the behavioral effects of transplantation. The results of these behavioral studies are numerically presented along with all other results in Table 1.

As previously described, P30 Pten mice exhibit abnormalities in social behavior and novel object exploration (Vogt et al., 2015). Consistent with these previous findings, we found that P50 shamtreated Pten mice also exhibited reduced social interaction when a novel juvenile mouse was introduced into their cages compared with their sham-treated wild-type littermates (Mann-Whitney test, $U=28.5$ and $p=0.0004 ; n=11$ sham-treated mutants and $n=20$ sham-treated wild-type; Fig. 2A). Surprisingly, interneuron transplantation rescued social interaction deficits in Pten mice by increasing recipient social interaction (Mann-Whitney test, $U=25.5$ and $p=0.01 ; n=12$ transplant recipient mutants; Fig. 2A). We did not observe a statistically significant difference 
Table 1. Compiled numerical results and statistics

Pten recipient behavior

$\begin{array}{lcc} & \text { Wild-type; sham, } & \text { Mutant; sham, } \\ & \text { mean } \pm \text { SEM } & \text { mean } \pm \text { SEM } \\ \text { Social interaction; duration, } s & 104.7 \pm 10.3 & 49.0 \pm 19.2 \\ & (n=20) & (n=11) \\ \text { Novel object exploration; duration, } \mathrm{s} & 14.7 \pm 2.1 & 9.8 \pm 2.0 \\ & (n=20) & (n=13) \\ \text { Elevated plus maze, entries into open arms } & 31.5 \pm 1.6 & 38.5 \pm 6.0 \\ \text { Elevated plus maze; time in open arms, } \mathrm{s} & (n=20) & (n=13) \\ & 140.2 \pm 16.2 & 176.5 \pm 35.7 \\ \text { Open field; time in center, } \mathrm{s} & (n=20) & (n=13) \\ & 54.5 \pm 6.6 & 37.0 \pm 6.0 \\ \text { Open field; distance traveled, } \mathrm{m} & (n=20) & (n=13) \\ & 54.7 \pm 3.6 & 95.4 \pm 10.1 \\ & (n=20) & (n=13)\end{array}$

Cd-1 recipient behavior

Social interaction; duration, $s$

Novel object exploration; duration, $s$

Elevated plus maze, entries into open arms

Elevated plus maze; time in open arms, $s$

Open field; time in center, $s$

Open field; distance traveled, $\mathrm{m}$
Sham; mean \pm SEM,

$n=16$ animals

$88.1 \pm 12.1$

$12.7 \pm 2.3$

$59.3 \pm 8.2$

$99.2 \pm 12.8$

$29.6 \pm 5.6$

$58.9 \pm 4.5$
Cells; mean \pm SEM,

$$
n=16 \text { animals }
$$

$77.7 \pm 10.4$

$15.1 \pm 1.9$

$53.5 \pm 3.8$

$154.7 \pm 20.0$

$31.3 \pm 2.7$

$66.8 \pm 5.8$
Mutant; cells, mean \pm SEM

$97.5 \pm 23.0$

$(n=12)$

$10.4 \pm 1.2$

$(n=12)$

$43.8 \pm 4.7$

$(n=12)$

$228 \pm 31.6$

$(n=12)$

$28.2 \pm 6.3$

$(n=12)$

$104.7 \pm 15.6$

$(n=12)$
Mann-Whitney $\quad \mathbf{0 . 0 0 0 4}$ (wild-type; sham vs mutant; sham) $\mathbf{0 . 0 1}$ mutant; sham vs mutant; cells

Mann-Whitney $\quad 0.07$ (wild-type; sham vs mutant; sham) 0.35 (mutant; sham vs mutant; cells)

Mann-Whitney $\quad 0.28$ (wild-type; sham vs mutant; sham) 0.44 (mutant; sham vs mutant; cells)

Mann-Whitney $\quad 0.60$ (wild-type; sham vs mutant; sham)

0.38 (mutant; sham vs mutant; cells)

Mann-Whitney $\quad \mathbf{0 . 0 4}$ (wild-type; sham vs mutant; sham)

0.26 (mutant; sham vs mutant; cells)

$\mathbf{0 . 0 0 0 8}$ (wild-type; sham vs mutant; sham) 0.57 (mutant; sham vs mutant; cells)

Slice recording from Pten recipient excitatory neurons

$\begin{array}{cc}\begin{array}{c}\text { Wild-type; Sham. } \\ \text { mean } \pm \text { SEM, }\end{array} & \text { Mutant; sham. } \\ n=17 \text { cells } & \text { mean } \pm \text { SEM, } \\ \text { from } 3 \text { animals } & \text { from } 3 \text { animals } \\ 4.2 \pm 0.3 & 5.4 \pm 2.2 \\ 23.8 \pm 0.9 & 22.8 \pm 1 \\ 5.8 \pm 0.2 & 5.8 \pm 0.2\end{array}$
Mutant; cells. mean \pm SEM,



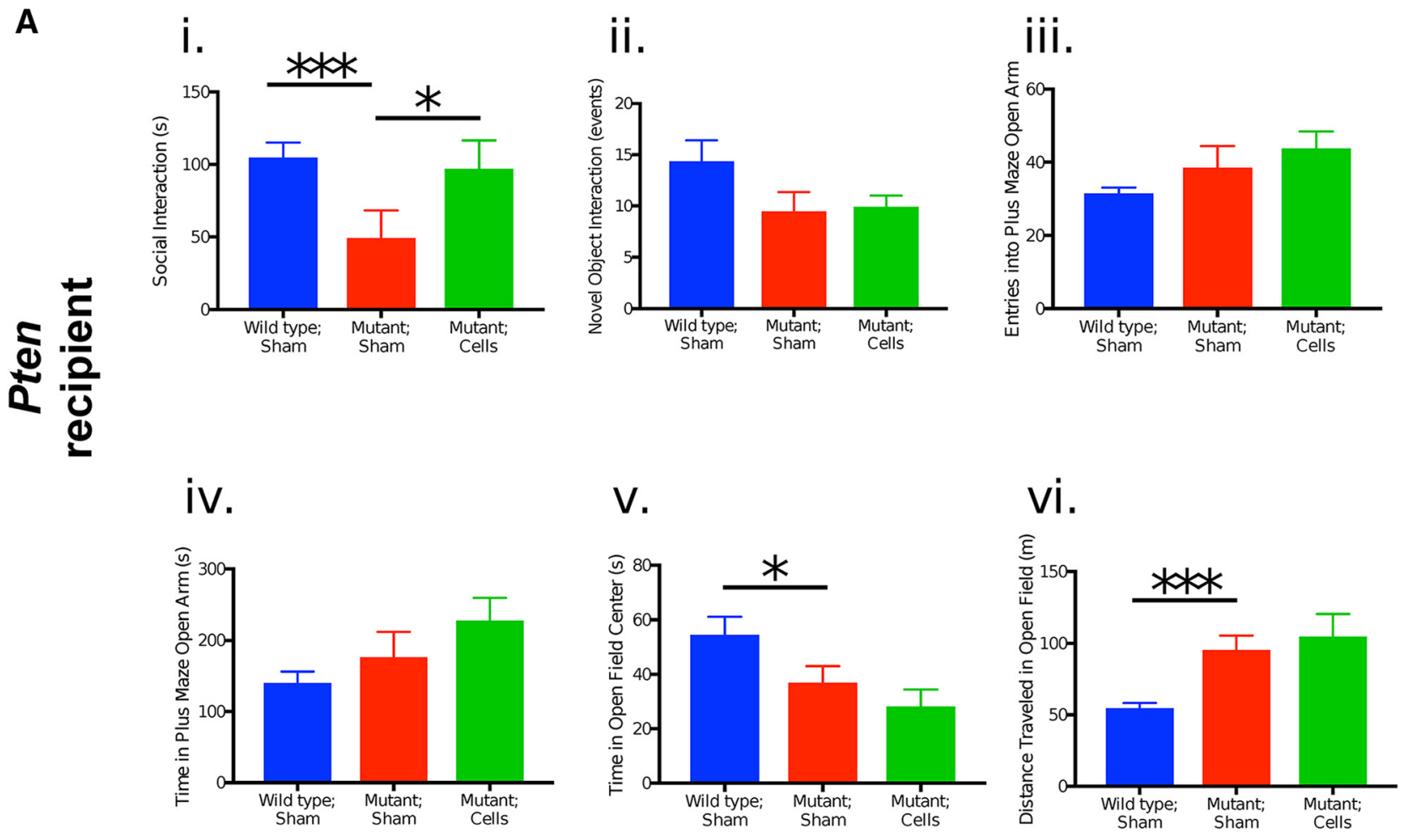

B

i.

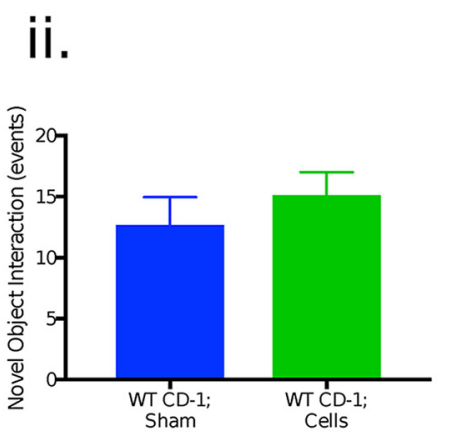

iii.
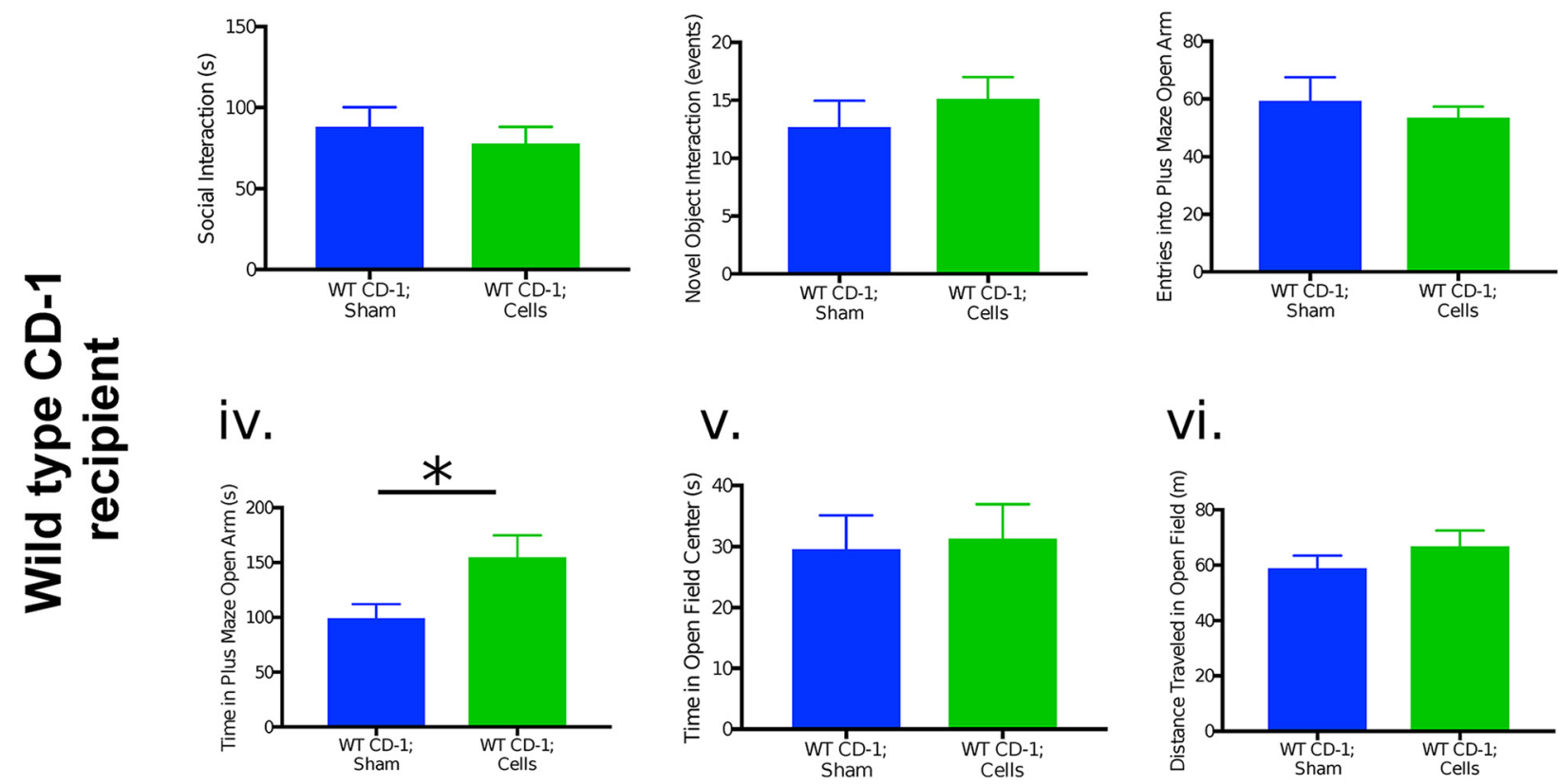

Figure 2. Interneuron transplantation exerts recipient-dependent behavioral effects, rescuing social interaction deficits in Pten mice. $A$, Behaviors of Pten mice and wild-type littermates that received interneuron precursor transplants (green) or sham, vehicle injections (blue and red). Ai, Social interaction. Aii, Novel object exploration. Aiii, Open arm entries in elevated plus maze. Aiv, Time spent in open arms in the elevated plus maze. Av, Time spent in center of open field. Avi, Distance traveled in open field. Data were analyzed using Mann-Whitney test. Graphs depict mean \pm SEM. $n=11-20$ animals per group. ${ }^{*} p<0.05,{ }^{* * *} p<0.001$. B, Same as $A$, but for wild-type CD-1 recipients (transplant recipient, green, $n=16$; sham recipient, blue). Data were analyzed using Mann-Whitney test. Graphs depict mean \pm SEM. $n=16$ animals per group. ${ }^{*} p<0.05$.

in social interaction behavior between transplant-recipient Pten mice and sham-treated wild-type littermates (Mann-Whitney test, $U=84.5$ and $p=0.17$ ). Sex differences were unlikely to account for the increased social interaction behavior of transplant recipient Pten mice compared with sham-treated Pten mice, as these groups were of relatively similar sex ratios (transplant recipient mutant, 7 male: 5 female; sham-treated mutant, 6 male: 5 female).

Whereas we previously observed reduced novel object exploration in P30 Pten mice (Vogt et al., 2015), here we observed a 
nonsignificant trend toward reduced novel object exploration in P50 sham-treated Pten mice compared with sham-treated wildtype mice (Mann-Whitney, $U=80.0$ and $p=0.07 ; n=13$ sham-treated mutants and $n=20$ sham-treated wild-type; Fig. $2 A)$. These differences may be related to the ages of the mice studied (P50 here vs P30 in previous study; Vogt et al., 2015) and/or to effects of the sham surgery. Interneuron transplantation did not significantly alter novel object exploration by Pten mice (Mann-Whitney test, $U=60.5$ and $p=0.35 ; n=12$ transplant recipient mutants; Fig. $2 A$ ). To determine whether transplantation increased Pten mutant social interaction through a nonspecific effect, which might involve a reduction in recipient anxiety phenotype or an increase in locomotor behavior, we also tested transplant recipient behavior in the elevated plus maze and open field. Consistent with prior findings from P30 Pten mice (Vogt et al., 2015), sham-treated Pten mice did not exhibit behavioral differences in the elevated plus maze (entries into open arms, time spent in open arms) compared with sham-treated wild-type littermates (Mann-Whitney test, entries into open arms, $U=100$ and $p=0.28$; time spent in open arms, $U=115$ and $p=0.60$, respectively; $n=13$ sham-treated mutants and $n=$ 20 sham-treated wild-type; Fig. 2A). Transplantation did not alter behavior of Pten mice in the elevated plus maze (entries into open arms, time spent in open arms) compared with sham treatment of Pten mice (Mann-Whitney test, entries into open arms, $U=63.5$ and $p=0.44$; time spent in open arms, $U=61$ and $p=$ $0.38 ; n=12$ transplant recipient mutants). Finally, unlike P30 Pten mice, which did not exhibit altered behavior in the open field (Vogt et al., 2015), sham-treated Pten mice (age $\sim$ P50) exhibited greater avoidance of the open field's center area (Mann-Whitney test, $U=73$ and $p=0.04$ ) and greater locomotor activity (total distance traveled in open field; Mann-Whitney test, $U=42.0$ and $p=0.0008)$ compared with sham-treated wild-type mice $(n=13$ sham-treated mutants and $n=20$ sham-treated wild-type). Interneuron transplantation did not alter these behavioral measures in Pten mice (Mann-Whitney test; open-field center time: $U=57.0$ and $p=0.26$; total distance traveled: $U=67.0$ and $p=$ $0.57 ; n=12$ transplant recipient mutants; Fig. $2 A$ ). Thus, interneuron transplantation rescued social interaction deficits in Pten mice without significantly altering other behavioral measures (exploration of a novel object, open field, or elevated plus maze).

\section{Interneuron transplantation exerts distinct behavioral effects in wild-type CD-1 mice}

We next studied the behaviors of P50 wild-type CD-1 mice that received immature interneuron transplants at $\mathrm{P} 1-\mathrm{P} 3$ into the PFC. These experiments allowed us to examine whether transplantation restored Pten recipient social behavior by specifically correcting or compensating for circuit abnormalities present in Pten mice, or if transplantation instead exerted a circuit effect that nonspecifically increases social interaction regardless of recipient type.

In contrast to its effects in Pten mice, interneuron transplantation did not significantly alter social interaction behavior of wild-type CD-1 recipient mice (Mann-Whitney test, $U=106.5$ and $p=0.43 ; n=16$ animals in each group; Fig. $2 B$ ). Moreover, whereas transplantation into Pten mice did not alter recipient behavior in the elevated plus maze (Fig. $2 A$ ), transplantation increased the time wild-type CD-1 recipients spent in the elevated plus maze open arms (Mann-Whitney test, $U=73.0$ and $p=$ $0.04 ; n=16$ animals in each group; Fig. $2 B$ ). Transplantation did not have a significant effect on the number of entries CD-1 recip- ients made into the elevated plus maze open arms (Mann-Whitney test, $U=114.0$ and $p=0.61 ; n=16$ animals in each group; Fig. 2B). Novel object exploration and open-field behavior of wild-type CD-1 recipient mice, were not significantly altered by transplantation (Mann-Whitney test; novel object exploration: $U=101.0$ and $p=0.32$; time spent in open field center: $U=$ 120.0 and $p=0.77$; total distance traveled in open field: $U=$ 101.0 and $p=0.32 ; n=16$ animals in each group; Figs. $2 B$ ). Thus, transplantation modified different behaviors in Pten mice (social interaction; Fig. $2 A$ ) compared with wild-type CD-1 mice (elevated plus maze exploration; $2 B$ ). Together, these findings indicate that transplantation elicits behavioral effects that vary with recipient background.

\section{Interneuron transplantation does not restore wild-type measures of spontaneous inhibitory synaptic signaling in Pten mice}

Even though Pten mice exhibit a $\sim 50 \%$ reduction in the number of MGE-derived cortical interneurons, the frequency of spontaneous inhibitory synaptic currents in layer $2 / 3$ pyramidal neurons is nearly doubled in this model (Vogt et al., 2015). To assess whether the rescue of social interaction deficits in Pten mice was associated with a restoration of wild-type levels of synaptic inhibition, we obtained voltage-clamp recordings in vitro from pyramidal neurons of the recipient medial PFC (Fig. 3A). Brain slices were prepared from transplant-recipient Pten mice (age $\sim$ P50; $n=15$ cells from 4 animals), sham-treated Pten mice ( $n=10$ cells from 3 animals), and sham-treated wild-type littermates ( $n=17$ cells from 3 animals). Figure $3 B$ depicts representative spontaneous inhibitory post-synaptic current (sIPSC) traces recorded from medial PFC layer 2/3 pyramidal neurons. Consistent with prior results (Vogt et al., 2015), sIPSC frequencies were higher in sham-treated Pten mice compared with their sham-treated wildtype littermates $\left(t_{(25)}=3, p=0.006\right.$ by $t$ test; Fig. $\left.3 D\right)$, whereas sIPSC amplitudes and half-widths did not statistically differ between these groups $\left(t_{(25)}=0.76, p=0.45\right.$ and $t_{(25)}=0.14, p=0.88$ by $t$ test, respectively; Fig. $3 C, E)$.

In prior studies, transplantation into wild-type recipients has consistently been found to increase sIPSC frequencies in recipient pyramidal neurons (Alvarez-Dolado et al., 2006; Baraban et al., 2009; Southwell et al., 2012). Here, transplantation into Pten recipient did not normalize elevated sIPSC frequencies in Pten mice; rather, it further increased sIPSC event frequencies $\left(t_{(23)}=\right.$ 2.4, $p=0.02$ by $t$ test; Fig. $3 D)$. Interneuron transplantation did not significantly alter sIPSC amplitudes in Pten mice $\left(t_{(23)}=1.9\right.$, $p=0.06$ by $t$ test; Fig. $3 D$ ), but did increase sIPSC half-widths $\left(t_{(23)}=3.2, p=0.004\right.$ by $t$ test; $\left.3 E\right)$.

We next examined whether transplanted interneurons altered synaptic inhibition onto native MGE-derived interneurons of Pten mice (Fig. $3 F$ ). We obtained voltage-clamp recordings from tdTomato $^{+}$(Nkx2.1-Cre;Ai14) native interneurons in layer $2 / 3$ of medial PFC in sham-treated ( $n=6$ cells from 2 animals) and transplant-recipient Pten mice ( $n=8$ cells from 3 animals). Representative sIPSC traces are depicted in Figure $3 G$. We observed no significant differences between the amplitudes $\left(t_{(12)}=0.16\right.$, $p=0.78$ by $t$ test; Fig. $3 H)$, frequencies $\left(t_{(12)}=0.04, p=0.97\right.$ by $t$ test; $3 I)$, or half-widths $\left(t_{(12)}=1, p=0.33\right.$ by $t$ test; $\left.3 J\right)$ of sIPSCs in native interneurons between sham-treated and transplantrecipient Pten mice.

Together, these data indicate that interneuron transplantation into Pten mice increased synaptic measures of inhibitory signaling onto recipient pyramidal neurons, but not interneurons. These results are similar to previous transplantation studies using 
A
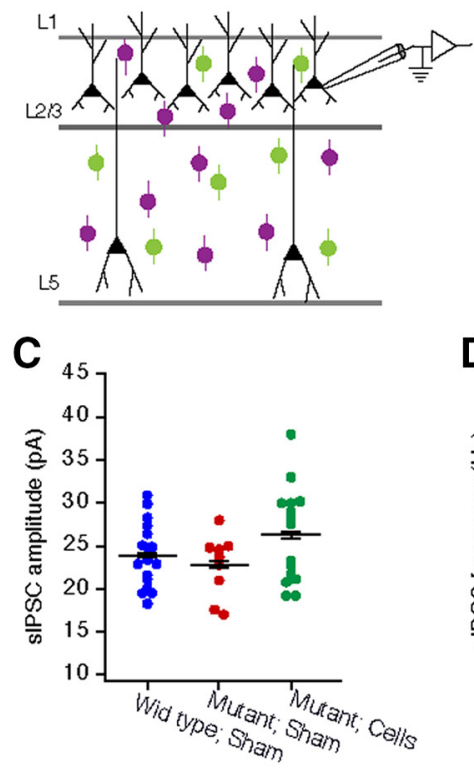

$\mathbf{F}$

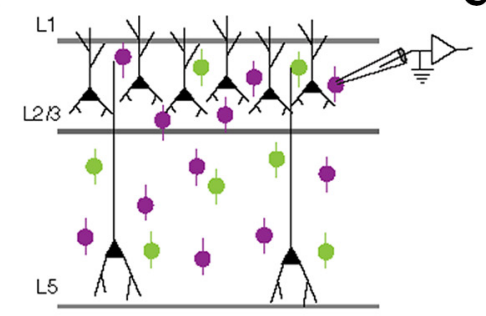

H

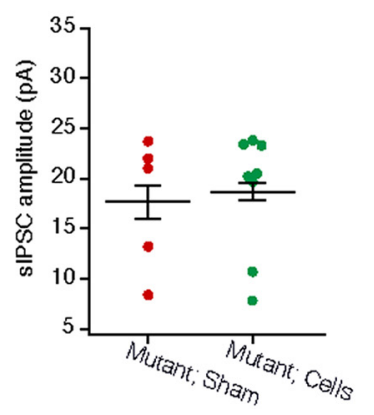

I
B

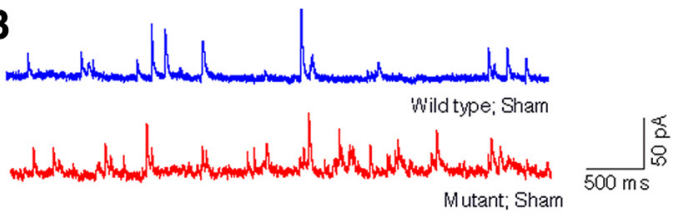

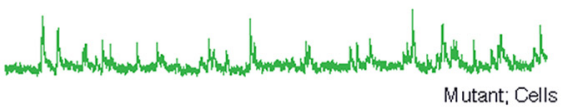

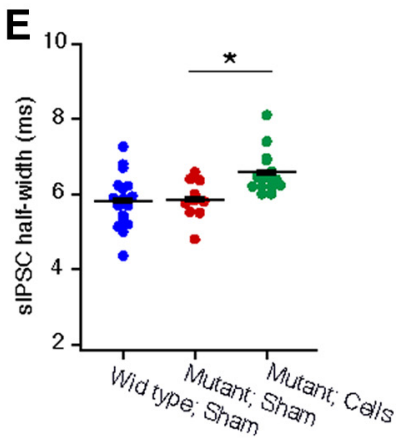

G
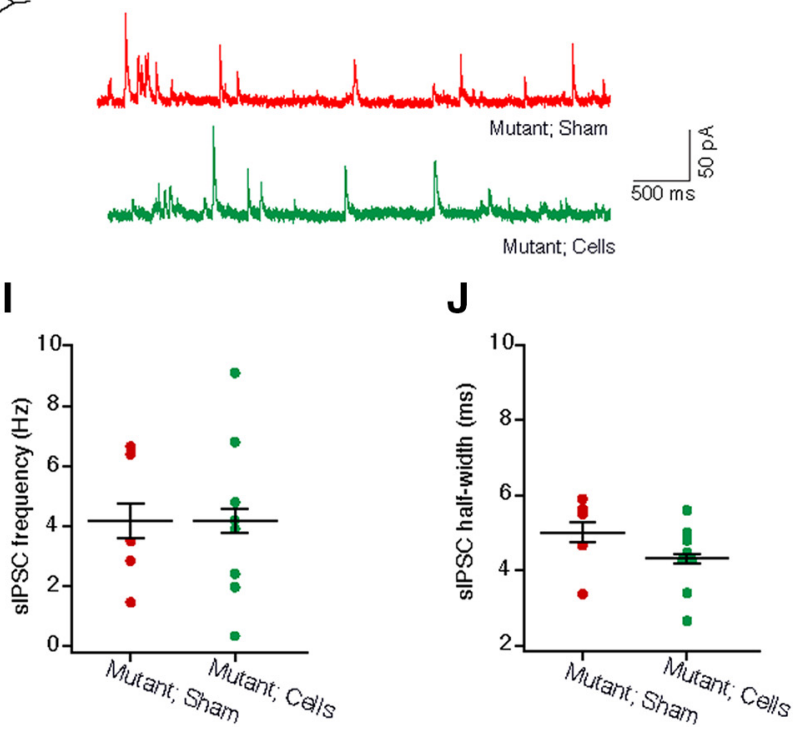

Figure 3. Interneuron transplantation into Pten mice does not restore in vitro measures of spontaneous inhibitory signaling to wild-type levels. $A$, Schematic, Patch-clamp recordings of recipient pyramidal neurons in brain slices. Voltage-clamp recordings were obtained from layer $(L) 2 / 3$ pyramidal neurons in medial PFC. Recipient pyramidal neurons were identified by morphology and the absence of GFP or tdTomato fluorescence; transplanted interneurons were identified by GFP expression; native, Nkx2.7-lineage interneurons were identified by tdTomato expression. $\boldsymbol{B}$, Representative traces showing sIPSCs from layer 2/3 pyramidal neurons in sham-treated wild-type littermates (blue), sham-treated Pten mice (red), and transplant-recipient Pten mice (green). C, layer 2/3 pyramidal neuron sIPSC amplitudes did not differ between sham-treated wild-type littermates (blue), sham-treated Pten mice (red), and transplant-recipient Pten mice (green). D, sIPSC event frequency was increased in sham-treated Pten mice (red) compared with sham-treated wild-type controls (blue). Interneuron transplantation into Pten mice (green) further increased sIPSC frequency. $\boldsymbol{E}$, Interneuron transplantation increased layer 2/3 pyramidal neuron sIPSC half-widths in Pten mice. $\boldsymbol{F}$, Schematic, Patch-clamp recordings from native $N k \times 2.7$-lineage interneurons. $\boldsymbol{G}$, Representative traces showing sIPSCS from native layer 2/3 Nkx2.7-lineage interneurons in sham-treated Pten mice (red), and transplant-recipient Pten mice (green). $\boldsymbol{H}-\boldsymbol{J}$, Native layer $2 / 3$ interneuron sIPSC mean amplitudes $(\boldsymbol{H})$, frequencies $(\boldsymbol{I})$ and half-widths $(\boldsymbol{J})$ did not differ between sham-treated (green) and transplant-recipient (red) Pten mice. Graphs depict mean \pm SEM; $n=$ $6-17$ cells per group. ${ }^{*} p<0.05$.

wild-type recipients in which transplanted MGE-derived interneurons increased synaptic inhibition onto recipient excitatory neurons without affecting inhibition onto interneurons (Alvarez-Dolado et al., 2006; Baraban et al., 2009; Southwell et al., 2012; Hsieh and Baraban, 2017). Thus, transplantation did not restore measures of synaptic inhibition toward wild-type levels in Pten mice; rather, it further increased spontaneous synaptic inhibition (which, in sham-treated Pten mice, was already elevated). As such, the rescue of social behavior in Pten mice is unlikely to occur through a mechanism that either involves, or is reflected by, a restoration of wild-type levels of synaptic inhibition.

\section{Transplantation does not alter in vivo baseline EEG activity} in Pten mice or wild-type CD-1 mice

In addition to assessing the effects of transplantation on measures of synaptic inhibition in vitro (Fig. 3), we also performed EEG measurements to examine its effects on recipient circuit activity in vivo (Fig. 4). Six weeks after transplantation, we placed EEG 
A WT; Sham
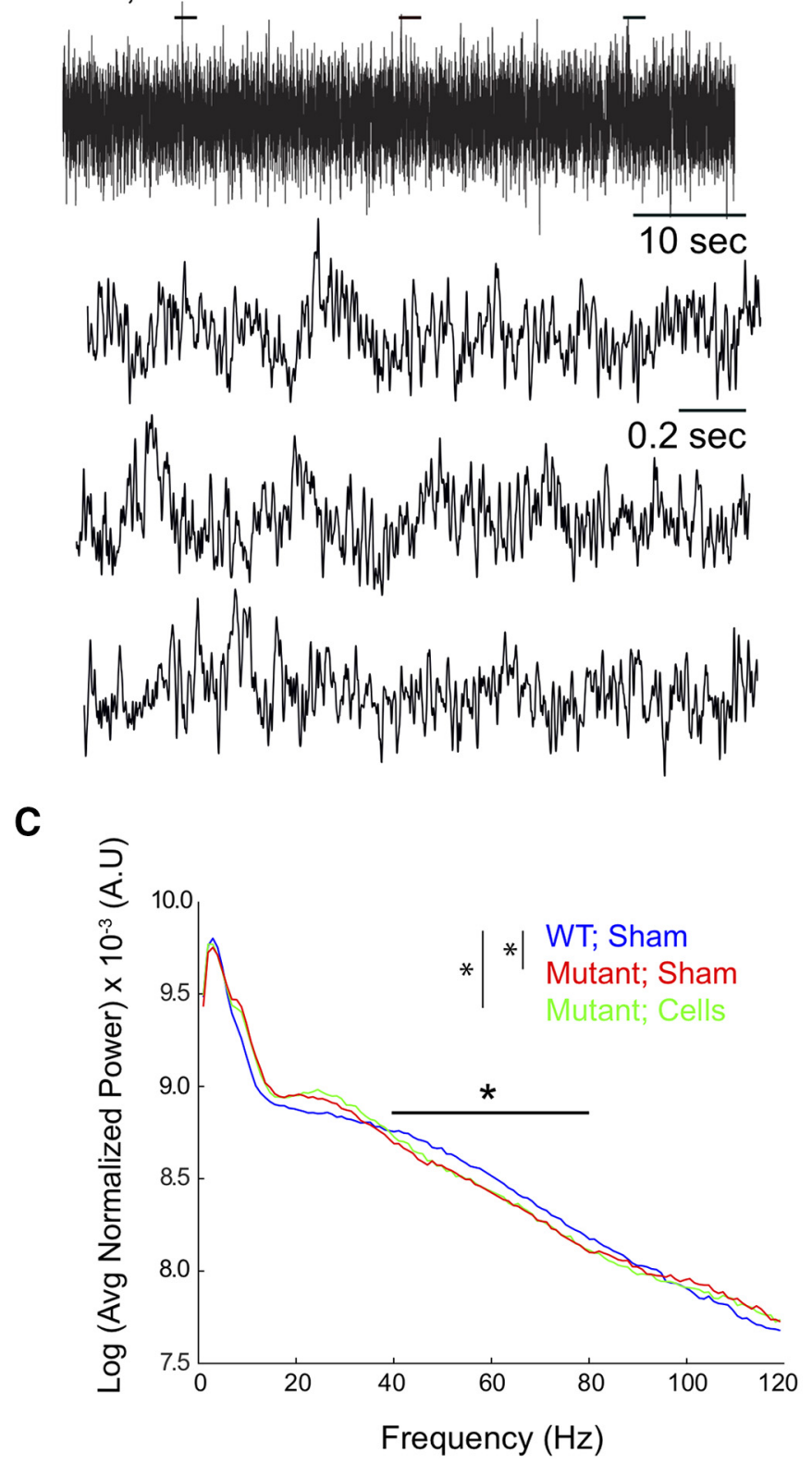

B Mutant; Sham
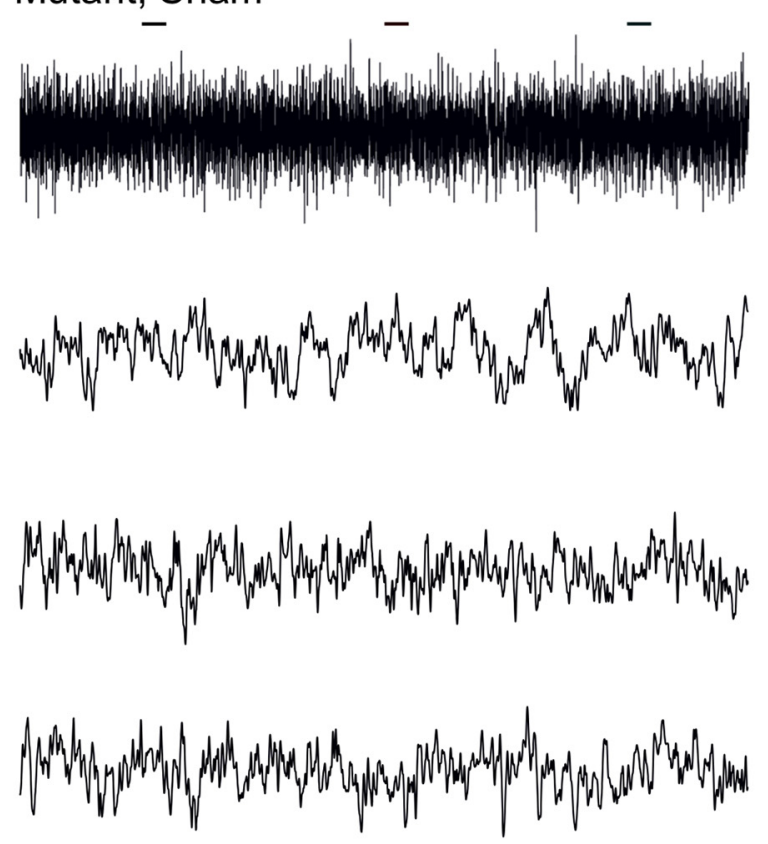

D

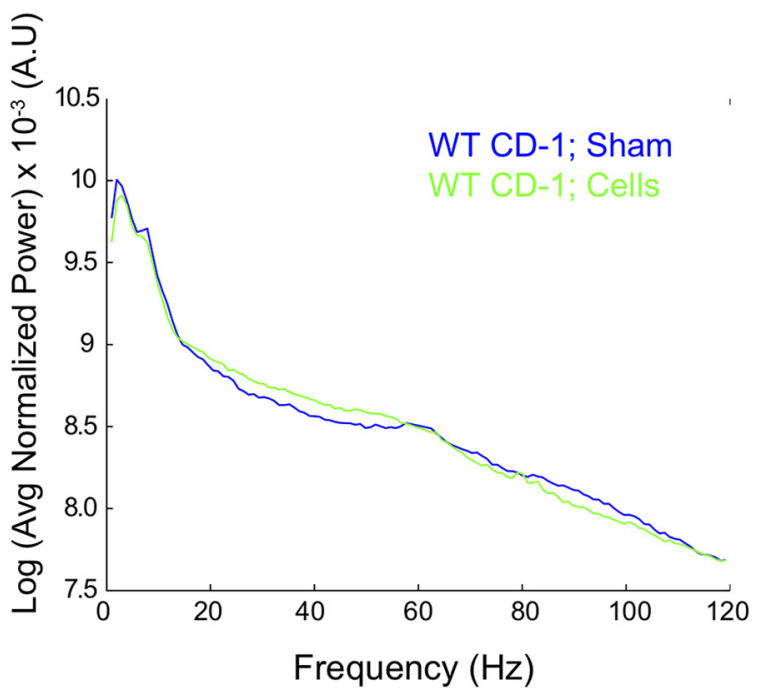

Figure 4. Interneuron transplantation does not affect baseline state oscillatory patterns in Pten mice or wild-type CD1 mice. A, Sample EEG recording from the medial PFC of a sham-treated wild-type control mouse (top). Expanded traces (bottom three) correspond to periods recorded in the top trace, as denoted by the horizontal bars. B, Sample EEG recording from the medial PFC of a sham-treated Pten mutant mouse (top). Expanded traces (bottom three) correspond to periods recorded in the top trace, as denoted by the horizontal bars. $C$, Plots depicting the average log transforms of baseline EEG power spectra from Pten mice, normalized by the total power from 0 to $120 \mathrm{~Hz}$ (excluding $58-62 \mathrm{~Hz}$ ). Sham-treated Pten mice (red) exhibited a decrease in EEG gamma power $(40-80 \mathrm{~Hz}$ ) compared with sham-treated wild-type littermates (blue). Transplantation did not alter baseline oscillatory patterns in Pten mice (green). $\boldsymbol{D}$, Plots depicting the average log transforms of baseline EEG power spectra from wild-type CD-1 recipient mice that received interneuron transplants (green) or sham injections (blue), normalized by the total power from 0 to 120 $\mathrm{Hz}$ (excluding $58-62 \mathrm{~Hz}$ ). Transplantation did not alter baseline EEG activity in wild-type CD-1 mice. Data in $\boldsymbol{C}$ and $\boldsymbol{D}$ were analyzed by $t$ test. $n=9-16$ per group. ${ }^{*} p<0.05$.

electrodes over the PFC of Pten mice and wild-type CD1 mice, and then performed baseline EEG measurements on isolated, freely behaving recipients 1 week after EEG placement.

As previously described, altered inhibitory synaptic signaling in P30 Pten mice is accompanied by a specific reduction of baseline EEG power in the gamma range (Vogt et al., 2015). EEG traces from wild-type and mutant animals were superficially similar (Fig. $4 A, B$ ), and we did not observe any overt signs of behavioral or electrographic seizures. Consistent with these prior findings, sham-treated P50 Pten mice exhibited reduced baseline EEG gamma $(40-80 \mathrm{~Hz})$ power compared with sham-treated wild-type littermates (Fig. $4 C ; t_{(19)}=2.7$ and $p=0.01$ by $t$ test; $n=9$ sham-treated mutants and $n=11$ sham-treated wild-type). Transplantation into the PFC also did not alter baseline gamma power in Pten mice (Fig. $4 C$; $t_{(15)}=0.3$ and $p=0.77$ by $t$ test; $n=$ 8 transplant-recipient mutants), nor did it affect baseline power in other frequency bands (e.g., $\beta, \alpha$, or $\theta ; p=0.55-0.74, t_{(15)}=$ $0.3-0.6$ by $t$ test). As a result, baseline EEG gamma power remained reduced in transplant-recipient Pten mice compared with sham-treated wild-type littermates $\left(t_{(18)}=2.4\right.$ and $p=0.03$ by $t$ test; $n=8$ transplant recipient mutant and $n=11$ shamtreated wild-type).

We likewise examined whether transplantation altered baseline EEG properties in wild-type CD-1 recipient mice. Across all 
A

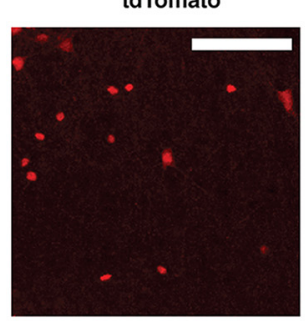

B

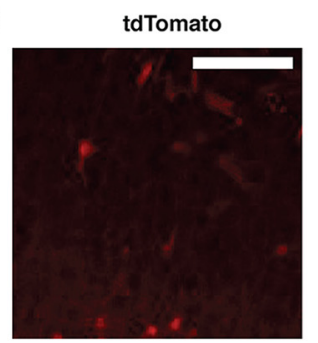

C

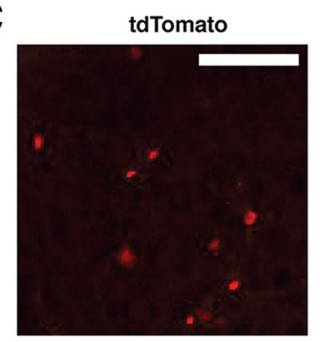

GFP

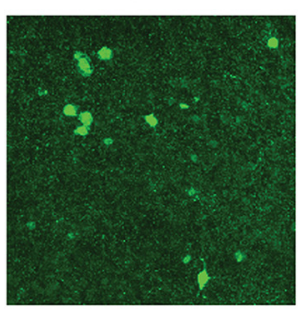

GFP

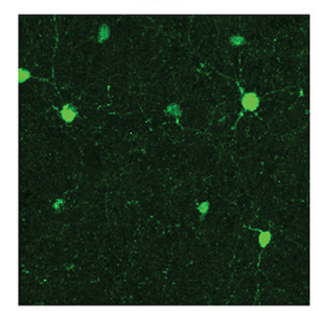

GFP

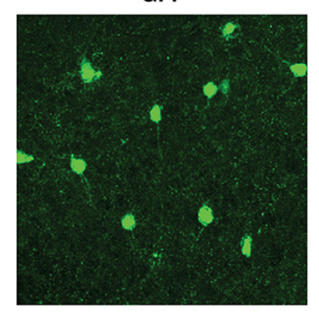

SST

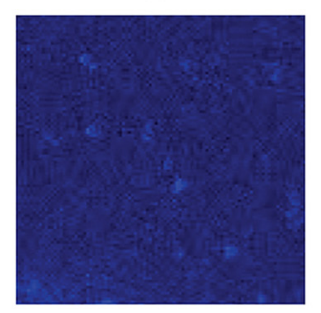

PV

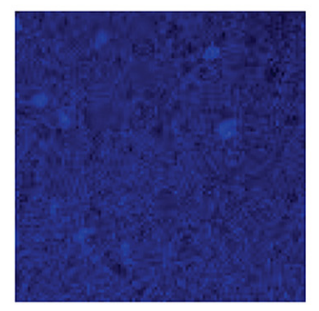

Reelin

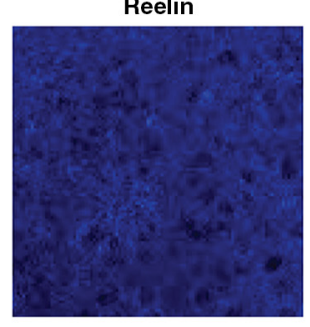

Merge

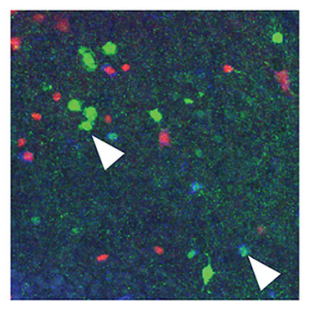

Merge

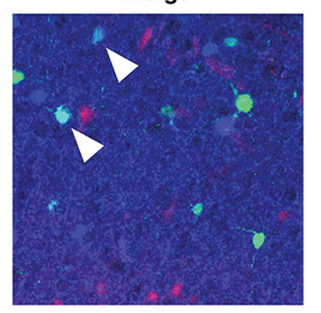

Merge

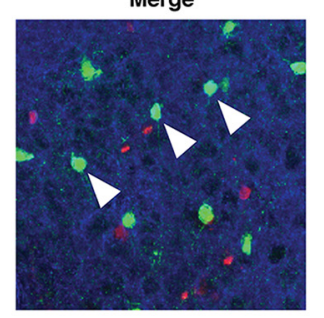

D

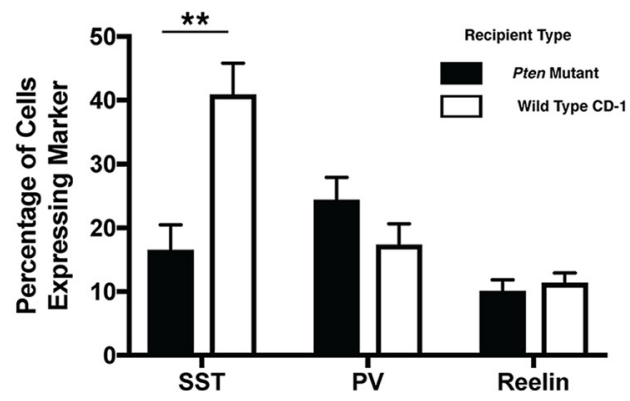

Figure 5. Recipient genotype affects the subtype composition of the surviving transplanted interneuron population. $\boldsymbol{A}$, Immunofluorescent staining of frontal cortical sections from P50 Pten mice that received interneuron transplants. Images depict tdTomato expression in native, $N k x$ - 2 . 1-lineage interneurons (red, far left), GFP expression in transplanted interneurons (green, near left), and SST expression (blue, near right). Merged images are depicted on far right; arrowheads depict colabeled transplanted cells. $\boldsymbol{B}$, Same as $\boldsymbol{A}$, but for PV expression. $\boldsymbol{C}$, Same as $\boldsymbol{A}$, but for Reelin expression. $D$, Quantification of marker expression by transplanted interneurons in Pten mice and wild-type CD-1 mice. When transplantation was performed into Pten mice (black) the SST-expressing transplanted population was specifically reduced in relative number compared with transplants performed into wild-type CD-1 mice (white). Scale bars: $\boldsymbol{A}-\boldsymbol{C}$, $150 \mu \mathrm{m}$. Graph depicts mean \pm SEM. Data in $\boldsymbol{D}$ were analyzed by Mann-Whitney test; $n=399-574$ cells per group. ${ }^{* *} p<0.01$.

bandwidths, we observed no significant difference in baseline oscillatory power between transplant recipient and sham-treated wild-type CD- 1 mice $t_{(25)}=0.26-1.49$ and $p=0.15-0.80$ by $t$ test; Fig. $4 B ; n=16$ transplant recipient and $n=11$ sham-treated animals). Thus, in both the Pten mutant and wild-type recipient backgrounds, transplantation did not modify baseline state EEG measures.

Recipient Pten background affects the subtype composition of surviving wild-type transplanted interneurons

After completing behavioral experiments, we performed histology to confirm the presence of GFP + transplanted cells in recipient mice (Fig. 5). By this time ( $\sim 8$ weeks after transplantation) transplanted cells were expected to have dispersed and differen- tiated into a complement of mature interneuron subtypes (Wonders and Anderson, 2005; Alvarez-Dolado et al., 2006; Miyoshi et al., 2010; Southwell et al., 2010). Transplanted cells were identified in PFC, as well as anterior cingulate, dorsal insular, primary motor, secondary motor, and somatosensory cortical areas. Transplanted cells were not noted in the olfactory bulb, hippocampus, or subcortical regions. To determine whether host Pten status contributes to the differentiation and/or survival of transplanted interneurons, we performed immunostaining to characterize transplanted cell molecular marker expression (Figs. 5A-C).

In previous studies of transplantation into wild-type recipients, the majority of surviving MGE-derived transplanted interneurons expressed either SST or PV, with a larger fraction of cells expressing SST (Alvarez-Dolado et al., 2006; Baraban et al., 
2009; Southwell et al., 2010). Consistent with these previous results, in wild-type CD-1 recipients, $40.9 \pm 4.9 \%$ of transplanted cells expressed SST ( $n=490$ cells analyzed), $17.4 \pm 3.3 \%$ expressed PV ( $n=499$ cells), and $11.4 \pm 1.6 \%$ expressed Reelin ( $n=474$ cells; $n=6$ animals; Fig. 5D). Because transplanted SST interneurons outnumbered transplanted PV interneurons in wild-type recipients, we expected that transplantation might at least partially restore the reduced SST-PV ratio present in Pten mice. Surprisingly, in Pten recipients, transplanted SST interneurons did not outnumber transplanted PV interneurons. In fact, only $16.3 \pm 3.9 \%$ of transplanted cells expressed SST $(n=574$ cells analyzed), whereas $24.4 \pm 3.5 \%$ expressed PV $(n=458$ cells), and $10.1 \pm 1.8 \%$ expressed Reelin ( $n=399$ cells; $n=5$ animals; Fig. 5D). Our findings indicate that recipient background (Pten mutant vs wild-type CD-1) exerted a non-cell autonomous effect on the subtype composition of the transplanted interneurons: the population of transplanted SST-expressing neurons was depleted in Pten recipients compared with wild-type CD- 1 recipients $(16 \pm 4 \%$ vs $41 \pm 5 \%$ in Pten mutant vs wildtype CD1 recipients; Mann-Whitney test, $U=1.0$ and $p=0.009$; Fig. 5D). It is unknown whether this resulted from decreased survival of SST-expressing cells, altered differentiation of precursors into SST versus PV interneurons, and/or altered expression of the SST phenotype.

\section{Discussion}

We have found that wild-type embryonic interneuron transplantation into the PFC of neonatal Pten mice rescued recipient social interaction deficits without normalizing elevated measures of synaptic inhibition in vitro or reduced baseline gamma band EEG activity in vivo. By contrast, in wild-type recipients, transplantation did not alter social behavior. Together our findings indicate that: (1) interneuron transplantation can rescue behavior even when recipient measures of synaptic inhibition are elevated, and (2) transplantation can rescue behavior without normalizing measures of circuit function (e.g., levels of synaptic inhibition and baseline state EEG signals).

Interneuron transplantation has been conceptualized as a method for increasing synaptic inhibition in recipient circuits (Alvarez-Dolado et al., 2006; Baraban et al., 2009; Southwell et al., 2012; Hsieh and Baraban, 2017), and, as such, it has typically been applied toward disease models characterized by increased cellular excitation or decreased inhibition. It is hypothesized that transplantation normalizes behavioral phenotypes by restoring excitatory/inhibitory balance (Southwell et al., 2014; Tyson and Anderson, 2014; Hunt and Baraban, 2015; Chohan and Moore, 2016; Spatazza et al., 2017). In the current study, however, we transplanted wild-type interneurons into Pten mice, a genetic model in which synaptic inhibition onto pyramidal neurons is elevated rather than diminished. Surprisingly, although transplantation improved social behavior deficits in Pten mice (Fig. 2), it further increased sIPSC frequencies in recipient pyramidal neurons, but not in recipient interneurons. Transplantation was thus able to modify a relevant disease phenotype (i.e., social behavior) in a model characterized by increased measures of synaptic inhibition, and it did so without normalizing this particular measure of circuit function. This has two important implications. First, it suggests that disease conditions characterized by reduced inhibition are not the only pathophysiologic backgrounds in which interneuron transplantation can yield therapeutic effects. Second, because transplantation rescued social interaction while further increasing abnormally elevated sIPSC frequencies in Pten mice, its effects here on recipient social behavior are unlikely to occur through a mechanism that involves, or is reflected by, a normalization of measures of synaptic inhibition.

Our observations raise the question of whether another measure of synaptic inhibition, such as evoked inhibition from a specific source onto a specific target, might be normalized by transplantation into Pten mice. Although this is certainly plausible, the combination of presynaptic inputs and postsynaptic cell types within PFC is extremely large, making a thorough investigation of this possibility nearly impossible in practice. Importantly, though, these considerations highlight a fundamental epistemological issue in this field: as noted in the Introduction, prior autism animal studies have relied on a small number of measurements of inhibitory function (e.g., the intrinsic firing properties of one interneuron subpopulation, sIPSC properties in one excitatory cell type, etc.) to hypothesize that inhibition is deficient in autism. These studies further demonstrated that experimental manipulations, such as benzodiazepine administration or optogenetic stimulation of interneurons, normalized behavior in the disease models, leading to the conclusion that autism-like behavior results from deficient inhibition and can be corrected by normalization of inhibition. While this is certainly straightforward and standard for the field, such an approach does not address whether other aspects of inhibitory circuit function may be normal or even excessive in the particular model, nor does it account for other potential, and more complex, circuit effects through which the manipulation may rescue behavior. Indeed, our results suggest that a simple measure of inhibition, such as sIPSC frequencies, does not necessarily capture how alterations of inhibition, or therapeutic manipulations of inhibition, relate to behaviorally relevant circuit function.

To begin to identify circuit measures that may be relevant to the behavioral effects of transplantation, we used EEG to examine emergent mesoscale aspects of recipient network function. Similar to its effects on sIPSCs, transplantation did not normalize decreased baseline EEG power in Pten mice. Of note, decreased baseline EEG power is consistent with an overall increase in circuit inhibition; in other mutant mouse models, defects in interneuron function are associated with elevated baseline EEG power (Cho et al., 2015), whereas optogenetic stimulation of excitatory neurons has been shown to increase local field potential gamma power (Yizhar et al., 2011). The fact that transplantation into Pten mice did not normalize sIPSCs or baseline EEG, suggests that transplantation elicits effects on circuit function that may not be captured by simple, static measures of circuit inhibition.

It is possible that transplantation altered social interaction, but not baseline EEG signals, because the transplanted population was specifically recruited during epochs of social interaction (Selimbeyoglu et al., 2017). Alternatively or in addition, transplanted interneurons may have yielded local circuit rearrangements through their effects on the plasticity of the recipient circuit (Southwell et al., 2010; Tang et al., 2014; Davis et al., 2015; Yang et al., 2016). These alterations, if present, may manifest during social interaction but not during baseline periods. In such a scenario, acute recruitment of the transplanted population during behavior may not be necessary to normalize recipient social behavior. Future studies might explore these possibilities by acutely silencing the transplanted population or measuring its activity during social interaction. Altogether, though, our findings indicate that interneuron transplantation did not exert behavioral effects by simply increasing inhibition in a nonspecific manner. Because transplanted interneurons dispersed both within and beyond the recipient PFC, we cannot conclude that 
our behavioral findings are due to transplant effects in the PFC, specifically.

Last, we found that the subtype composition of the transplanted population differed between Pten and wild-type CD-1 recipients. In wild-type $\mathrm{CD}-1$ recipients, the ratio of transplanted SS- to PV-expressing interneurons (i.e., the SST-PV ratio) was found to be $\sim 2.4: 1$ (Fig. 5D). In Pten mice, however, the SST-PV ratio of the surviving population was $\sim 0.7: 1$. Because the SST-PV ratio of endogenous, MGE-derived interneurons is $\sim 0.7: 1$ in Pten mice (Vogt et al., 2015), it is unlikely that transplantation corrected the imbalanced SST-PV ratio in Pten recipient cortex. Regardless, our findings suggest that recipient Pten function exerted a non-cell autonomous effect on the development of the transplanted population. As mentioned, the SST-PV ratio of transplanted interneurons may be reduced in Pten mice because of decreased survival of transplanted SST-expressing cells, reduced expression of SST by surviving cells, or both. Previous studies describing the activity-dependent maturation of SST phenotypes (De Marco García et al., 2011; Denaxa et al., 2012; Tuncdemir et al., 2016) suggest that abnormal neural circuit function in Pten mice could fail to drive SST expression in wild-type transplanted interneurons. The mechanistic basis for this effect remains to be addressed in future studies. Because transplanted cells were pooled from multiple embryonic donors and collected from the entire dorsoventral axis of the MGE (Wonders et al., 2008), it is unlikely that they differed with respect to interneuron subtype composition at the time of transplantation.

The varied compositions of the transplanted interneuron populations may explain why Pten mice and wild-type CD-1 mice exhibited divergent behavioral responses to transplantation. For example, a prior study using transplant populations enriched for either SST- or PV-expressing subtypes has described donor celltype-specific effects on recipient social interaction (Donegan et al., 2017). Future studies are likely to explore how transplanted interneurons differentially contribute to social behavior, synaptic physiology and mesoscale circuit function by measuring these features following the transplantation of specific subtypes of cells. Future studies may also examine whether transplantation alters social interaction phenotypes in recipients of other genetic backgrounds.

Our findings have number of implications for translational research. Because the subtype compositions of transplanted interneurons and their behavioral effects differed between wildtype recipient mice and Pten mice, future studies should not be designed under the assumption that transplanted interneurons develop and function similarly in wild-type settings and disease settings. Our findings also indicate that transplanted interneurons may not impact recipient circuit function or behavior simply by increasing inhibition in a static, global, or nonspecific fashion. Rather, or in addition, transplanted interneurons may modify network activity in a dynamic and behaviorally specific manner. As a result, assays performed in vitro, or during baseline states in vivo, may not always predict or capture the physiologic and/or behavioral effects of interneuron transplantation. Furthermore, transplantation may produce therapeutic effects by inducing novel neurophysiologic states, rather than simply restoring mutant circuits to a "wild-type" condition. Together, a better understanding of these processes will help to optimize transplantation strategies for clinical purposes.

\section{References}

Alvarez-Dolado M, Calcagnotto ME, Karkar KM, Southwell DG, Jones-Davis DM, Estrada RC, Rubenstein JL, Alvarez-Buylla A, Baraban SC (2006)
Cortical inhibition modified by embryonic neural precursors grafted into the postnatal brain. J Neurosci 26:7380-7389.

Baraban SC, Southwell DG, Estrada RC, Jones DL, Sebe JY, Alfaro-Cervello C, García-Verdugo JM, Rubenstein JL, Alvarez-Buylla A (2009) Reduction of seizures by transplantation of cortical GABAergic interneuron precursors into Kv1.1 mutant mice. Proc Natl Acad Sci U S A 106:15472-15477.

Cao W, Lin S, Xia Q, Du Y, Yang Q, Zhang M, Lu Y, Xu J, Duan SM, Xia J, Feng G, Xu J, Luo JH (2018) Gamma oscillation dysfunction in mPFC leads to social deficits in neuroligin 3 R451C knockin mice. Neuron 97:1253-1260.e7.

Cho KK, Hoch R, Lee AT, Patel T, Rubenstein JL, Sohal VS (2015) Gamma rhythms link prefrontal interneuron dysfunction with cognitive inflexibility in Dlx5/6+/- mice. Neuron 85:1332-1343.

Chohan MO, Moore H (2016) Interneuron progenitor transplantation to treat CNS dysfunction. Front Neural Circuits 10:64

Davis MF, Figueroa Velez DX, Guevarra RP, Yang MC, Habeeb M, Carathedathu MC, Gandhi SP (2015) Inhibitory neuron transplantation into adult visual cortex creates a new critical period that rescues article inhibitory neuron transplantation into adult visual cortex creates a new critical period that rescues impaired vision. Neuron 86:1055-1066.

De Marco García NV, Karayannis T, Fishell G (2011) Neuronal activity is required for the development of specific cortical interneuron subtypes. Nature 472:351-355.

De Rubeis S, He X, Goldberg AP, Poultney CS, Samocha K, Cicek AE, Kou Y, Liu L, Fromer M, Walker S, Singh T, Klei L, Kosmicki J, Shih-Chen F, Aleksic B, Biscaldi M, Bolton PF, Brownfeld JM, Cai J, Campbell NG, et al. (2014) Synaptic, transcriptional and chromatin genes disrupted in autism. Nature 515:209-215.

Denaxa M, Kalaitzidou M, Garefalaki A, Achimastou A, Lasrado R, Maes T, Pachnis V (2012) Maturation-promoting activity of SATB1 in MGEderived cortical interneurons. Cell Rep 2:1351-1362.

Donegan JJ, Tyson JA, Branch SY, Beckstead MJ, Anderson SA, Lodge DJ (2017) Stem cell-derived interneuron transplants as a treatment for schizophrenia: preclinical validation in a rodent model. Mol Psychiatry 22:1492-1501.

Geisheker MR, Heymann G, Wang T, Coe BP, Turner TN, Stessman HA, Hoekzema K, Kvarnung M, Shaw M, Friend K, Liebelt J, Barnett C, Thompson EM, Haan E, Guo H, Anderlid BM, Nordgren A, Lindstrand A, Vandeweyer G, Alberti A, et al. (2017) Hotspots of missense mutation identify novel neurodevelopmental disorder genes and functional domains. Nat Neurosci 20:1043-1051.

Gogolla N, Takesian AE, Feng G, Fagiolini M, Hensch TK (2014) Article sensory integration in mouse insular cortex reflects GABA circuit maturation. Neuron 83:894-905.

Hadjantonakis AK, Gertsenstein M, Ikawa M, Okabe M, Nagy A (1998) Generating green fluorescent mice by germline transmission of green fluorescent ES cells. Mech Dev 76:79-90.

Han S, Tai C, Westenbroek RE, Yu FH, Cheah CS, Potter GB, Rubenstein JL, Scheuer T, de la Iglesia HO, Catterall WA (2012) Autistic-like behaviour in Scnla1/2 mice and rescue by enhanced GABA-mediated neurotransmission. Nature 489:385-390.

Howard MA, Baraban SC (2016) Synaptic integration of transplanted interneuron progenitor cells into native cortical networks. J Neurophysiol 116:472-478.

Howard MA, Rubenstein JL, Baraban SC (2014) Bidirectional homeostatic plasticity induced by interneuron cell death and transplantation in vivo. Proc Natl Acad Sci U S A 111:492-497.

Hsieh JY, Baraban SC (2017) Medial ganglionic eminence progenitors transplanted into hippocampus integrate in a functional and subtypeappropriate manner. eNeuro 4:ENEURO.0359-16.2017.

Hunt RF, Baraban SC (2015) Interneuron transplantation as a treatment for epilepsy. Cold Spring Harb Perspect Med 5:a022376.

Iossifov I, Levy D, Allen J, Ye K, Ronemus M, Lee YH, Yamrom B, Wigler M (2015) Low load for disruptive mutations in autism genes and their biased transmission. Proc Natl Acad Sci U S A 112:E5600-E5607.

Jung EM, Moffat JJ, Liu J, Dravid SM, Gurumurthy CB, Kim W (2017) Arid $1 \mathrm{~b}$ haploinsufficiency disrupts cortical interneuron development and mouse behavior. Nat Neurosci 20:1694-1707.

Larimer P, Spatazza J, Stryker MP, Alvarez-Buylla A, Hasenstaub AR (2017) Development and long-term integration of MGE-lineage cortical interneurons in the heterochronic environment. J Neurophysiol 118:131-139. 
Lewis DA, Hashimoto T, Volk DW (2005) Cortical inhibitory neurons and schizophrenia. Nat Rev Neurosci 6:312-324.

Madisen L, Zwingman TA, Sunkin SM, Oh SW, Zariwala HA, Gu H, Ng LL, Palmiter RD, Hawrylycz MJ, Allan R, Lein ES, Zeng H (2010) A robust and high-throughput cre reporting and characterization system for the whole mouse brain. Nat Neurosci 13:133-140.

Marín O (2012) Interneuron dysfunction in psychiatric disorders. Nat Rev Neurosci 13:107-120.

Miyoshi G, Hjerling-Leffler J, Karayannis T, Sousa VH, Butt SJ, Battiste J, Johnson JE, Machold RP, Fishell G (2010) Genetic fate mapping reveals that the caudal ganglionic eminence produces a large and diverse population of superficial cortical interneurons. J Neurosci 30:1582-1594.

Roak BJ, Vives L, Fu W, Egertson JD, Stanaway IB, Phelps IG, Carvill G, Kumar A, Lee C, Ankenman K, Munson J, Hiatt JB, Turner EH, Levy R, O’Day DR, Krumm N, Coe BP, Martin BK, Borenstein E, Nickerson DA, et al. (2012) Multiplex targeted sequencing identifies recurrently mutated genes in autism spectrum disorders. Science 338:1619-1622.

Selimbeyoglu A, Kim CK, Inoue M, Lee SY, Hong ASO, Kauvar I, Ramakrishnan C, Fenno LE, Davidson TJ, Wright M, Deisseroth K (2017) Modulation of prefrontal cortex excitation/inhibition balance rescues social behavior in CNTNAP2-deficient mice. Sci Transl Med 9:eaah6733.

Southwell DG, Froemke RC, Alvarez-Buylla A, Stryker MP, Gandhi SP (2010) Cortical plasticity induced by inhibitory neuron transplantation. Science 327:1145-1148.

Southwell DG, Paredes MF, Galvao RP, Jones DL, Froemke RC, Sebe JY, Alfaro-Cervello C, Tang Y, Garcia-Verdugo JM, Rubenstein JL, Baraban SC, Alvarez-Buylla A (2012) Intrinsically determined cell death of developing cortical interneurons. Nature 491:109-113.

Southwell DG, Nicholas CR, Basbaum AI, Stryker MP, Kriegstein AR, Rubenstein JL, Alvarez-Buylla A (2014) Interneurons from embryonic development to cell-based therapy. Science 344:1240622.

Spatazza J, Mancia Leon WR, Alvarez-Buylla A (2017) Transplantation of GABAergic interneurons for cell-based therapy. Prog Brain Res 231:57-85.

Suzuki A, Yamaguchi MT, Ohteki T, Sasaki T, Kaisho T, Kimura Y, Yoshida R,
Wakeham A, Higuchi T, Fukumoto M, Tsubata T, Ohashi PS, Koyasu S, Penninger JM, Nakano T, Mak TW (2001) T cell-specific loss of Pten leads to defects in central and peripheral tolerance. Immunity 14:523-534.

Tanaka DH, Toriumi K, Kubo K, Nakajima K, Nabeshima T (2011) GABAergic precursor transplantation into the prefrontal cortex prevents phencyclidine-induced cognitive deficits. J Neurosci 31:1411614125.

Tang Y, Stryker MP, Alvarez-Buylla A, Espinosa JS (2014) Cortical plasticity induced by transplantation of embryonic somatostatin or parvalbumin interneurons. Proc Natl Acad Sci U S A 111:18339-18344.

Tuncdemir SN, Wamsley B, Stam FJ, Osakada F, Goulding M, Callaway EM, Rudy B, Fishell G (2016) Early somatostatin interneuron connectivity mediates the maturation of deep layer cortical circuits. Neuron 89:521-535.

Tyson JA, Anderson SA (2014) GABAergic interneuron transplants to study development and treat disease. Trends Neurosci 37:169-177.

Vogt D, Cho KKA, Lee AT, Sohal VS, Rubenstein JL (2015) The parvalbu$\mathrm{min} /$ somatostatin ratio is increased in Pten mutant mice and by human PTEN ASD alleles. Cell Rep 11:944-956.

Wonders CP, Anderson SA (2005) Cortical interneurons and their origins. Neuroscientist 11:199-205.

Wonders CP, Taylor L, Welagen J, Mbata IC, Xiang JZ, Anderson SA (2008) A spatial bias for the origins of interneuron subgroups within the medial ganglionic eminence. Dev Biol 314:127-136.

Xu Q, Tam M, Anderson SA (2008) Fate mapping Nkx2. 1-lineage cells in the mouse telencephalon. J Comp Neurol 506:16-29.

Yang W, Liu T, Cao J, Chen X, Liu X, Wang M, Su X, Zhang S, Qiu BL, Hu WX, Liu LY, Ma L, Yu YC (2016) Fear erasure facilitated by immature inhibitory neuron transplantation. Neuron 92:1352-1367.

Yizhar O, Fenno LE, Prigge M, Schneider F, Davidson TJ, O'Shea DJ, Sohal VS, Goshen I, Finkelstein J, Paz JT, Stehfest K, Fudim R, Ramakrishnan C, Huguenard JR, Hegemann P, Deisseroth K (2011) Neocortical excitation/inhibition balance in information processing and social dysfunction. Nature 477:171-178. 